\title{
Karl Popper 120th anniversary; Some reflections on the decision-making process in medicine
}

\author{
Elisha Krasin ${ }^{1}$ \\ ${ }^{1}$ Tel Aviv Sourasky Medical Center
}

March 30, 2021

\begin{abstract}
Rereading Popper's "The Logic of scientific discovery", at his 120th anniversary, brings some thoughts regarding the diagnostic process and decision making in medicine from the viewpoint of the classical scientific method. In recent years physicians are increasingly becoming technical experts who base their decision-making on uniform criteria, guidelines and classifications but unfortunately have moved away from understanding the basic concepts in the philosophy of science. This raises an ethically and philosophically important issue; what does a medical diagnosis mean? Is this an absolute or a relative truth? The implications of this question are enormous in terms of prognosis and treatment. Both patients and physicians should be educated about the nature of the diagnostic process.
\end{abstract}

\section{Karl Popper $120^{\text {th }}$ anniversary;}

\section{Some reflections on the decision-making process in medicine}

Running title: Some reflections on the decision-making process in medicine

Elisha Krasin MD

Division of Orthopedic Surgery, Tel Aviv Sourasky Medical Center, affiliated with the Faculty of Medicine, Tel Aviv University, Tel Aviv, Israel.

Correspondence: Elisha Krasin MD, https://orcid.org/0000-0001-7263-4422,

Email: ekrasin@outlook.co.il, Telephone: +972-3-6973484

Funding - No funding was received for this paper.Conflicts of interest/Competing interests - The author declared no conflict of interest.

Ethics approval - Not applicable.

Consent to participate - Not applicable.

\section{Abstract:}

Rereading Popper's "The Logic of scientific discovery", at his $120^{\text {th }}$ anniversary, brings some thoughts regarding the diagnostic process and decision making in medicine from the viewpoint of the classical scientific method. In recent years physicians are increasingly becoming technical experts who base their decisionmaking on uniform criteria, guidelines and classifications but unfortunately have moved away from understanding the basic concepts in the philosophy of science. This raises an ethically and philosophically 
important issue; what does a medical diagnosis mean? Is this an absolute or a relative truth? The implications of this question are enormous in terms of prognosis and treatment. Both patients and physicians should be educated about the nature of the diagnostic process.

Keywords: diagnostic criteria; diagnostic process; differential diagnosis; intent-to-treat analysis; predictive values; scientific method

\section{Main text:}

Inexperienced physicians tend to see diagnoses as absolute. It is more prominent among surgeons. One who sees during an operation, a nerve root that is compressed by a herniated disc or the collapse of the femoral head due to avascular necrosis, would be convinced that he has a good understanding of the pathophysiology of the disease and the causes that brought the patient to his attention. Unfortunately the situation is not always that clear. Rheumatic patients often spend weeks, months or even years before getting a final diagnosis. Incomplete lupus erythematosus (latent or "non-criteria" lupus) is a typical example. In oncology, the pathologist is not always convinced about the precise type of the tumor or even if the tumor is benign or malignant. Simple count of mitoses, that is prone to subjectivity, ${ }^{1}$ sometimes is the only method that determines if chemotherapy is necessary. The purpose of this paper is to examine the diagnostic process from the viewpoint of the classical scientific method, based on the work of Karl Popper, and to shed some light on the meaning of making a diagnosis.

Karl Popper was born in Vienna in 1902 to a converted Jewish family. After the annexation of Austria by the Nazis, he immigrated to New Zealand and later to Britain. He wrote several books that had a profound influence on the philosophy of science and medicine in the previous century. In his 1959 book "The Logic of scientific discovery" ${ }^{2}$ Popper has thoroughly analyzed the scientific method. He claims that after a tentative creation of a hypothesis that is "not justified in any way"; the hypothesis is tested by the way of "empirical applications of the conclusions that can be derived from it". A positive experiment is always temporary as any future negative experiment can overthrow the theory. In the process of medical diagnosis, when the patient presents with a symptom, the physician makes a hypothetic list of diagnoses that can possibly explain the symptom. The next stage would be to exclude or prove the diagnoses by the means of history taking, physical examination and different laboratory and imaging tests. In the same manner as Popper described, a proof or a disproof of a diagnosis is in effect only until a new test will change the conclusion. Reproducibility of an experiment is another condition for the effect to be scientifically significant ${ }^{2}$ and certainly is important in the diagnostic process. Multiple repetitions of an experiment bring us closer to exemplary proof, which is actually unreachable, as a single negative experiment can disprove the theory. An experiment actually measures the probability of a result and almost never the p-value in the medical field would be zero. Even the most successful drug or procedure sometimes fails, and patients in the control group can recover with placebo treatment. Intent-to-treat analysis necessitates including dropouts in the final statistic, thus decreasing the final effect of the test, drug or the procedure that is under experiment. ${ }^{3}$ Each medical laboratory test or imaging modality has its own sensitivity, specificity and positive and negative predictive values that are never absolute. So actually even at the end of the diagnostic process we remain with a hypothesis that is proved both temporarily and in a probabilistic manner. Ilgen et $\mathrm{al}^{4}$ indicate that skillful clinicians work comfortably when uncertain and acquiring this ability to act in complex settings is necessary for trainees.

Another subject that is a source of confusion and misunderstanding is the common use of diagnostic criteria. They are very useful, especially for the less experienced physician, but one should always keep in mind that diagnostic criteria cannot be more accurate than the tests they are based upon, prior to the clinical validation of the criteria for diagnosis. For example, the diagnostic criteria for a prosthetic joint infection rely on cultures, histology, elevation of acute phase reactants and some other tests. Each of these tests has its own sensitivity and specificity and the combination of tests does not create $100 \%$ specific and sensitive method. Eventually validation of each set of clinical criteria for diagnosis is based on a "gold standard test" that also has its sensitivity and specificity that never equals one. Overdependence on diagnostic criteria will eventually lead to misdiagnosis. For example, Li et al had found that the number of patients diagnosed with a prosthetic joint infection in a group, grossly varies depending on the diagnostic criteria that were used. ${ }^{5}$ 
In conclusion, the clinical data should be analyzed without prejudice. Flexibility and readiness to re-evaluate the conclusions when new information arrives is necessary. Criteria and classifications are intended only to be an aid in diagnosis, not the absolute truth. Understanding the intricacies of the diagnostic process is absolutely necessary to provide proper care.

References:

1. Balkenhol MCA, Tellez D, Vreuls W, et al. Deep learning assisted mitotic counting for breast cancer. Lab Invest . 2019;99(11):1596-1606. doi:10.1038/s41374-019-0275-0

2. Popper KR. The Logic of Scientific Discovery . Repr. 2008 (twice). Routledge; 2008.

3. Brody T. Chapter 8 - Intent-to-Treat Analysis Versus Per Protocol Analysis. In: Brody T, ed. Clinical Trials (Second Edition) . Academic Press; 2016:173-201. doi:10.1016/B978-0-12-804217-5.00008-4

4. Ilgen JS, Eva KW, de Bruin A, Cook DA, Regehr G. Comfort with uncertainty: reframing our conceptions of how clinicians navigate complex clinical situations. Adv Health Sci Educ Theory Pract . 2019;24(4):797809. doi:10.1007/s10459-018-9859-5

5. Li C, Renz N, Trampuz A, Ojeda-Thies C. Twenty common errors in the diagnosis and treatment of periprosthetic joint infection. Int Orthop . 2020;44(1):3-14. doi:10.1007/s00264-019-04426-7 